Рыбина О. В..", Шавель Ю. А.', Петренко А. А. ${ }^{2}$, Галайко М. В.', Литвиненко М. С.', Егорков В. Е.', Губкин А. В.'

$14 У 3$ «Центральная клиническая больница «РЖД-Медицина», 129128, Москва, Россия

2ГБУЗ г. Москвы Городская клиническая больница им. С. П. Боткина Департамента здравоохранения г. Москвы, 125284, Москва, Россия

\title{
PEЗЮME
}

Введение. Множественная миелома (ММ) и хронический миелолейкоз (ХМЛ) - два гематологических заболевания, возникающие вследствие опухолевой трансформации лимфоидной и миелоидной клеток-предшественниц и не имеющие общую клетку-предшественницу. Оба заболевания крайне редко встречаются у одного больного.

Цель - описать клиническое наблюдение больной, у которой через несколько месяцев после начала лечения ММ был выявлен ХМЛ.

Основные сведения. Представлено клиническое наблюдение диагностики ММ и ХМЛ у одной больной в возрасте 62 лет. Первично диагностирована MM, проведено 5 курсов по программе VD. В связи свыраженной полинейропатией лечение было прекращено. Больная была переведена на поддерживающую терапию талидомидом. Через 12 месяцев от начала терапии талидомидом был диагностирован XMЛ BCR-ABL (p190) и BCR-ABL (p210). Проводилась терапия иматинибом, с кратковременным эффектом, в дальнейшем переведена на терапию дазатинибом. Через 16 месяцев от начала терапии дазатинибом диагностирован рецидив ММ и прогрессия ХМЛ.

Ключевые слова: множественная миелома, хронический миелолейкоз, ингибиторы тирозинкиназы, химиотерапия, лучевая терапия

Конфликт интересов: авторы заявляют об отсутствии конфликта интересов.

Финансирование: исследование не имело спонсорской поддержки.

Благодарность: авторы выражают благодарность коллективу клинико-диагностической лаборатории ЧУЗ «Центральная клиническая больница «РЖД-Медицина» и сотрудникам ФГБУ «Национальный медицинский исследовательский центр гематологии» Министерства здравоохранения Российской Федерации за помощь в проведении исследования.

Для цитирования: Рыбина О.В., Шавель Ю.А., Петренко А.А., Галайко М.В., Литвиненко М.С., Егорков В.Е., Губкин А.В. Развитие множественной миеломы и хронического миелолейкоза у одной больной. Гематология и трансфузиология. 2020; 65(4): 501-513. https://doi.org/10.35754/02345730-2020-65-4-501-513 


\section{COEXISTENCE OF MULTIPLE MYELOMA AND CHRONIC MYELOLEUKOSIS IN ONE PATIENT}

Rybina O. V. ${ }^{\prime *}$, Shavel Yu.A. ', Petrenko A. A. ${ }^{2}$, Galaiko M. V.', Litvinenko M. S.', Egorkov V. E.', Gubkin A. V.'

'Central Clinical Hospital "RZD-Medicine", 129128, Moscow, Russian Federation

${ }^{2}$ Botkin Moscow City Hospital, 125284, Moscow, Russian Federation

\section{ABSTRACT}

Intoduction. Multiple myeloma (MM) and chronic myelogenous leukaemia (CML) are two haematological malignancies developing through tumour transformation of lymphoid and myeloid progenitor cells, respectively, not sharing a common ancestry. Coexistence of the two diseases is extremely rare.

Aim. Clinical description of a patient diagnosed with CML in a few months after start of $M M$ therapy.

Main findings. We report a clinical case of $M M$ and $C M L$ in a 62 years-old female patient. MM was diagnosed newly and followed by 5 VD chemotherapy cycles. Treatment discontinued due to severe polyneuropathy. The patient was transferred to thalidomide maintenance therapy. CML was diagnosed 12 months after initiation of thalidomide therapy: $B C R-A B L(p 190)$, $B C R-A B L$ (p210). Since imatinib produced short-term effect, dasatinib therapy was started. Following 16 months after the onset of dasatinib therapy, MM relapse and CML progression were diagnosed.

Keywords: multiple myeloma, chronic myeloid leukaemia, tyrosine kinase inhibitors, chemotherapy, radiation therapy

Conflict of interest: the authors declare no conflict of interest.

Financial disclosure: the study had no sponsorship.

Acknowledgements: the authors are grateful to the team of the Clinical and Diagnostic Laboratory at the Central Clinical Hospital "RZD-Medicine" and staff of the National Research Center for Hematology, Ministry of Health of the Russian Federation, for help in conducting research.

For citation: Rybina O.V., Shavel Yu.A., Petrenko A.A., Galaiko M.V., Litvinenko M.S., Egorkov V.E., Gubkin A.V. Coexistence of multiple myeloma and chronic myeloleukosis in one patient. Russian Journal of Hematology and Transfusiology (Gematologiya i transfuziologiya). 2020; 65(4): 501-513 (in Russian). https:// doi.org/10.35754/0234-5730-2020-65-4-501-513

\section{Введение}

Множественная миелома (ММ) - злокачественное лимфопролиферативное заболевание, морфологическим субстратом которого являются атипичные плазматические клетки, секретирующие моноклональный иммуноглобулин. Заболеваемость ММ в России в 2017 г. составила 2,8 на 100 тыс. населения, возрастная медиана заболеваемости - 63,7 года [1]. По оценкам Американского онкологического общества, за последнее десятилетие показатели заболеваемости ММ существенно не изменились; по прогнозам в 2020 г. в США будут диагностированы 32270 новых случаев MM (17 $530-$ у мужчин и $14740-$ у женщин). Риск заболеть в течение жизни составляет 1/132 (0,76 \%) [2].
Хронический миелолейкоз (ХМЛ) - миелопролиферативное заболевание, возникающее в результате приобретенной хромосомной транслокации $(9 ; 22)$ (филадельфийская хромосома) в плюрипотентной стволовой клетке. Частота встречаемости в 2017 г. составила 1-2 случая на 100 тыс. взрослого населения [3]. По данным Американского онкологического общества [2], у 1 из 526 человек в течение жизни будет установлен диагноз ХМЛ (0,19 \%). Медиана возраста при установке заболевания составляет около 64 лет.

Возникновение одновременно обоих заболеваний у одного человека - крайне редкое событие, так как обе патологии возникают вследствие опухолевой 
трансформации различных гемопоэтических клеточных линий и не имеют общую клетку-предшественницу [4]. Генез возникновения MМ и ХМЛ у одного человека в настоящее время остается до конца неясным, однако существует несколько гипотез, пытающихся объяснить данный феномен [5-9].

Цель работы - описать клиническое наблюдение больной, у которой через несколько месяцев после начала лечения ММ был выявлен ХМЛ.

\section{Клиническое наблюдение}

Больная Л., 62 лет, впервые госпитализирована в отделение гематологии ЦКБ № 2 им. Н. А. Семашко в июле 2016 г. с жалобами на выраженный болевой синдром в пояснично-крестцовом отделе позвоночника, в ребрах. Перед госпитализацией в амбулаторных условиях ей выполнено иммунохимическое исследование белков сыворотки крови и мочи. В сыворотке крови патологических градиентов не выявлено, однако выявлены гипогаммаглобулинемия (иммуноглобулин G - 67 Ед/л, иммуноглобулин M - 43 Ед/л, иммуноглобулин A - 18 Ед/л), $\beta_{2}$-микроглобулин 2,63 мг/л, криоглобулины не обнаружены. В моче выявлен белок Бенс-Джонса $\mathrm{k}-0,9$ г/л (2,02 г/сут). Ранее больная наблюдалась по месту жительства в связи с псориазом, осложненным псориатическим артритом; дегенеративным сочетанным аортально-митральным пороком сердца (комбинированный порок аортального клапана с преобладанием стеноза, умеренная недостаточность митрального клапана).

При обследовании в ЦКБ № 2 им. Н. А. Семашко в клиническом анализе крови выявлены анемия до 102 г/л, увеличение скорости оседания эритроцитов (СОЭ) до 41 мм/час. Концентрации лейкоцитов и тромбоцитов крови находились в пределах референсных значений, формула крови была без изменений. При биохимическом исследовании крови обнаружены гиперкальциемия (кальций - 2,69 ммоль/мкл), признаки почечной недостаточности, при этом не было гиперпротеинемии и гипоальбуминемии. В пунктате костного мозга выявлено 6 \% плазматических клеток (рис. 1), при гистологическом исследовании костного мозга межбалочные пространства были широкие, деятельный костный мозг преобладал над жировым и был представлен всеми тремя ростками кроветворения, обнаружены множественные плазмоцитоидные клеточные элементы, в том числе двуядерные, лежавшие изолированно и формировавшие обширные скопления. По результатам рентгенологического обследования выявлены признаки деструктивных изменений в обеих плечевых костях, переломы в третьем и четвертом ребрах слева. Деструктивной патологии костей черепа и таза не выявлено. По данным магнитно-резонансной томографии позвоночника обнаружены компрессионные переломы $\mathrm{Th}_{\mathrm{XII}}$ и $\mathrm{L}_{\mathrm{I}}$ позвонков (рис. 2). На основании результатов проведенного обследования был

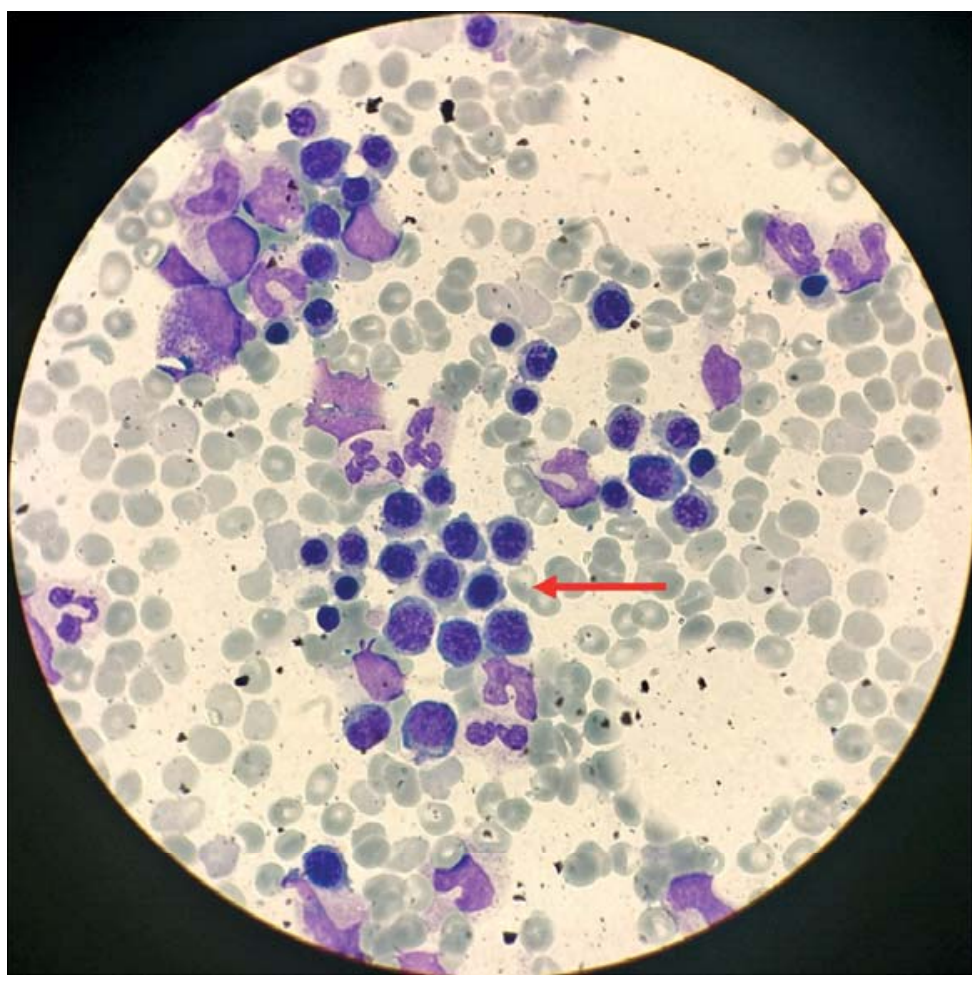

Рисунок 1. Пунктат костного мозга с увеличенным количеством атипичных плазматических клеток. Окраска гематоксилин-эозином, увеличение $\times 100$

Figure 1. Bone marrow punctate with increased population of atypical plasmacytes. Haematoxylin-eosin, $\times 100$

установлен диагноз: «Множественная миелома, протекающая с протеинурией Бенс-Джонса k, распространенным остеодеструктивным процессом - IIA стадия (Durie - Salmon) [1], I стадия (ISS) [1], осложненная компрессионными переломами $\mathrm{Th}_{\mathrm{XII}}$ и $\mathrm{L}_{\mathrm{I}}$ позвонков, переломами третьего и четвертого ребер слева».

Больной проведена лучевая терапия (суммарная очаговая доза - 20 Гр) на область $\mathrm{Th}_{\mathrm{XII}}-\mathrm{L}_{\mathrm{I}}$ позвонков и начата терапия по программе VD (бортезомиб 1,3 мг/ м²/сут, дексаметазон 40 мг/сут в 1-й, 4-й, 8-й и 11-й дни терапии). После 4 курсов лечения белок Бенс-Джонса в моче не определялся, констатирована иммунохимическая ремиссия. В то же время после проводимой терапии появились и постепенно нарастали снижение чувствительности кожи ладоней и подошв, судороги в икроножных мышцах, боли в проекции стоп и кистей, сохранявшиеся в покое и ночное время. Диагностирована периферическая полинейропатия II-III степени по NCI СТC [10]. Больной было начато лечение витаминами группы $\mathrm{B}$, прегабалином и $\alpha$-липоевой кислотой, а также проведен пятый курс по программе VD. Однако явления полинейропатии сохранялись, в связи с чем было принято решение прервать химиотерапию.

С ноября 2016 г. в течение 12 месяцев проводилась терапия талидомидом в дозе 100 мг/сут. В ноябре 2017 г., т. е. через 17 месяцев после установления диагноза ММ, при обследовании было констатировано, что сохранялась ремиссия ММ (белок Бенс-Джонса в моче не определялся, концентрация свободных легких цепей (СЛЦ) в сыворотке крови была в пределах 

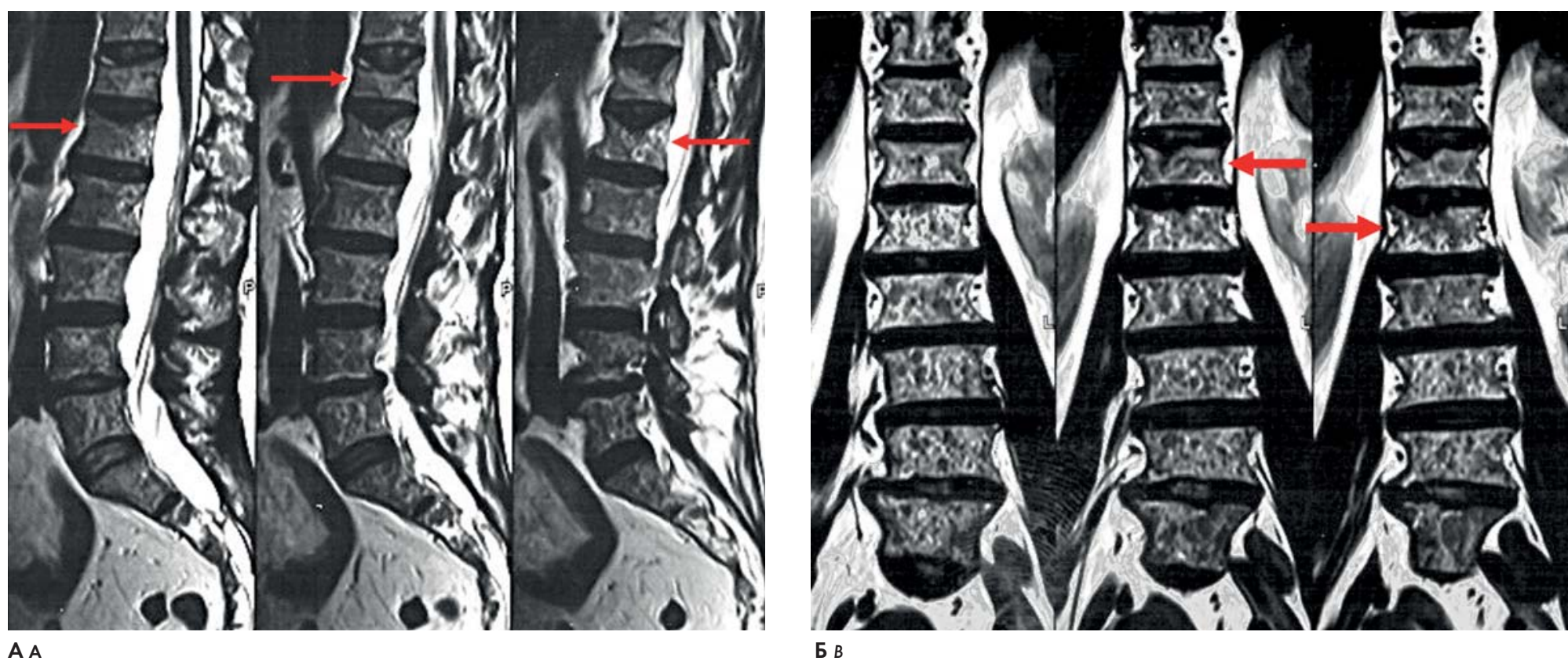

A A

Б B

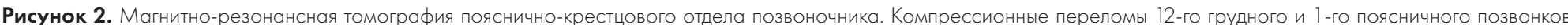
(указаны стрелками). А - сагиттальная проекция; Б - фронтальная проекция

Figure 2. Magnetic resonance imaging of lumbosacral spine. Compression fractures of

нормы, соотношение $\mathrm{k} / \lambda$-СЛЦ не нарушено), но в гемограмме впервые появились лейкоцитоз $\left(50,8 \times 10^{9} /\right.$ л), тромбоцитопения $(24 \times 10 \%$ /л), концентрация гемоглобина составила 123 г/л, СОЭ - 8 мм/час. В формуле крови отмечен сдвиг влево до бластных форм (бластные клетки - 2 \%, миелоциты - 10 \%, метамиелоциты - 6 \%, палочкоядерные нейтрофилы - $14 \%$, сегментоядерные нейтрофилы - 44 \%, эозинофилы $1 \%$, базофилы - 2 \%, лимфоциты - 12 \%, моноциты $-9 \%)$. В биохимическом анализе крови выявлено повышение концентрации лактатдегидрогеназы сыворотки до 1224 Ед/л; гиперпротеинемия, гиперкальциемия, почечная функция была не нарушена. В миелограмме бластные клетки составили 2,5 \%, пунктат был гиперклеточный с расширенным гранулоцитарным ростком. Плазматические клетки в пунктате костного мозга не обнаружены (рис. 3). С целью уточнения диагноза больной была выполнена трепанобиопсия костного мозга. При гистологическом исследовании трепанобиоптата костные балки были утолщены за счет напластования остеоида на эндост, костномозговые полости широкие, в них - резко гиперклеточный относительно возрастной нормы костный мозг, что было обусловлено расширением гранулоцитарного ростка. Гранулоцитарный росток был с дефицитом созревания, признаками нарушения зональности клеток зрелого и промежуточного пулов, представлен элементами промежуточного пула, вне зон эндоста были видны разрозненно расположенные элементы с незрелой/ бластной морфологией. Зрелые клетки определялись в уменьшенном количестве, были видны скопления клеток эозинофильной генерации. Больной было выполнено цитогенетическое исследование клеток пе- риферической крови, по результатам которого выявлена транслокация t(9;22)(q34;q11.2). Флуоресцентная гибридизация in situ (Fluorescence in situ hybridization, FISH) показала наличие в 17,68 \% клеток химерного гена $B C R-A B L(\mathrm{p} 190)$ и в 89,73\% - химерного гена $B C R-A B L$ (р210). При ультразвуковом исследовании гепатоспленомегалии и другой патологии органов брюшной полости не обнаружили. На основании результатов исследований у больной был диагностирован Ph-позитивный ХМЛ, хроническая фаза. С января 2018 г. начата терапия иматинибом в редуцированной в связи с тромбоцитопенией дозе 400 мг/сут. При обследовании в апреле 2018 г. уровень $B C R-A B L(\mathrm{p} 210)$ снизился до 9,62 \%, химерный ген $B C R-A B L$ (p190) не определялся. Ph-хромосома при стандартном цитогенетическом исследовании костного мозга не выявлялась. Диагностирован полный цитогенетический ответ. Однако в результате терапии ингибитором тирозинкиназы (ИТК) сохранялась стойкая тромбоцитопения (минимально $24 \times 10 \%$ ), требовавшая проведения заместительной терапии концентратами тромбоцитов. Остальные показатели крови оставались в пределах нормальных значений. При этом не было признаков прогрессии МM, сохранялась иммунохимическая ремиссия заболевания.

При обследовании в январе 2019 г. сохранялась тромбоцитопения 36-43 × 10\%/л. По данным FISH, в костном мозге уровень химерного гена $B C R-A B L(\mathrm{p} 210)$ составил $2,1 \%$, вновь появился химерный ген $B C R-A B L(\mathrm{p} 190)-$ уровень 0,04 \%. Выполнен мутационный анализ гена $B C R-A B L$ (р210), при котором мутации не обнаружены.

В январе 2019 г. больная была переведена на терапию дазатинибом в дозе 140 мг/сут. При обследовании 
в мае 2019 г. уровень химерного гена $B C R-A B L$ (p210) снизился до $0,171 \%, B C R-A B L$ (p190) не определялся. $\mathrm{Ph}$-хромосома не выявлена. Сохранялась умеренная тромбоцитопения $56-78 \times 10^{9} /$ л.

В сентябре 2019 г. при обследовании отмечено уменьшение $B C R-A B L$ (р210) до 0,064 \%, остальные показатели были без динамики. У больной был диагностирован большой молекулярный ответ и продолжена терапия дазатинибом. По данным иммунохимического исследования крови и мочи, в сентябре 2019 г. секреции парапротеина и белка Бенс-Джонса не выявлено, сохранялась иммунохимическая ремиссия ММ.

В январе 2020 г. появилась и прогрессивно наросла слабость, появилась одышка. При рентгенографии органов грудной клетки выявлены правосторонний гидроторакс до переднего отрезка 4-го ребра, диффузное уплотнение легочной ткани средней доли, в левом легком очаговых и инфильтративных изменений не было. Выполнена плевральная пункция, эвакуировано 450 мл транссудата. Ситуация была расценена как осложнение терапии дазатинибом, доза дазатиниба была уменьшена до 70 мг в день. По результатам рентгенографии органов грудной клетки в феврале и марте 2020 г. сохранялся базальный правосторонний гидроторакс без тенденции к нарастанию.

При обследовании в апреле 2020 г. в гемограмме концентрации лейкоцитов, эритроцитов, гемоглобина оставались в пределах нормальных значений, сохранялась изолированная тромбоцитопения $(63-70 \times 10 \%$ л). По данным иммунохимического исследования, в моче выявлен белок Бенс-Джонса k, концентрация СЛЦ в сыворотке составила 625 мг/л, с мочой выделялось 0,16 г/сут, имелась вторичная гипогаммаглобулинемия. По данным гистологического исследования костного мозга, костный мозг был гиперклеточный, имелась выраженная интерстициально-очаговая межтрабекулярная и паратрабекулярная инфильтрация зрелыми плазматическими клетками; миелопоэз был представлен тремя ростками: гранулоцитарный росток в умеренном количестве, представлен клеточными элементами разной степени зрелости, эритроидный росток был сужен, представлен мелкими скоплениями эритрокариоцитов нормобластического ряда; мегакариоциты немногочисленны, располагались неравномерно разрозненно, межтрабекулярно, обычного и небольшого размера с полиморфными ядрами. При молекулярно-цитогенетическом исследовании в $5 \%$ клеток была выявлена транслокация (9;22). Таким образом, у больной диагностированы рецидив ММ и прогрессия ХМЛ. В настоящее время проводится обследование, после чего будет принято решение о выборе тактики терапии.

\section{Обсуждение}

ММ и ХМЛ редко встречаются у одного больного. Подобная клиническая ситуация представляет интерес с точки зрения терапии и прогноза выживаемости

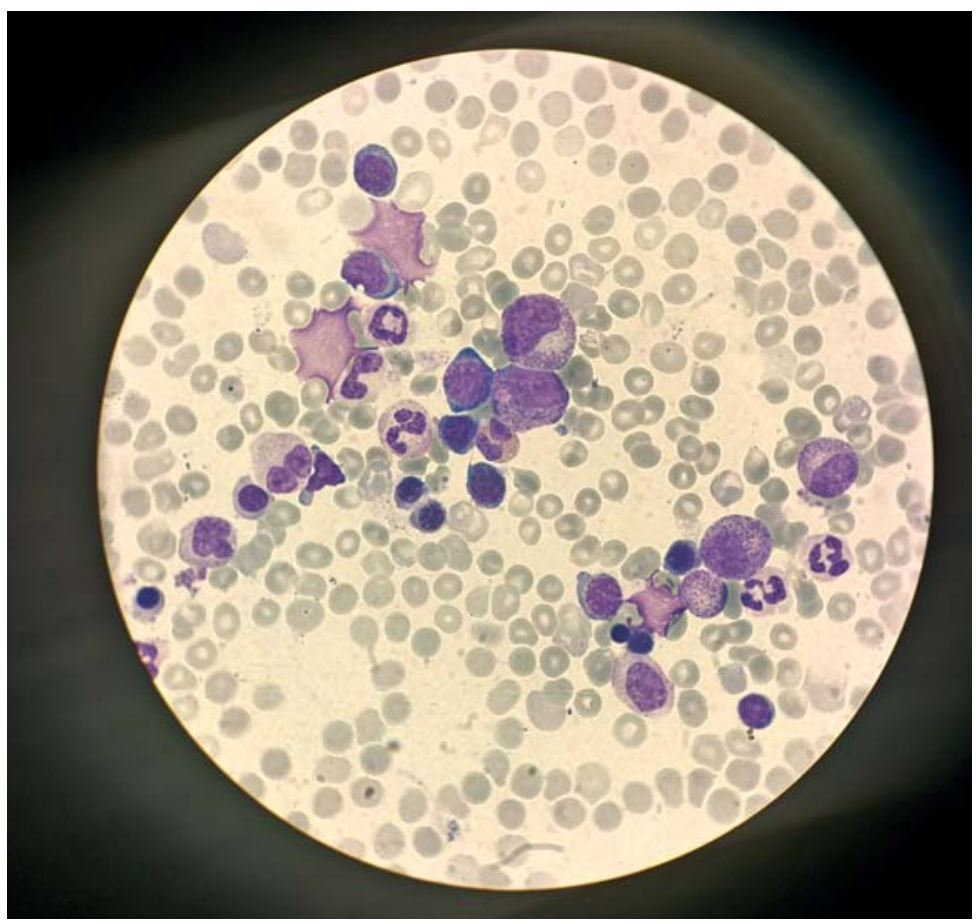

Рисунок 3. Пунктат костного мозга к моменту установления хронического миелолейкоза. Окраска гематоксилин-эозином, увеличение × 100

Figure 3. Bone marrow punctate at diagnosis of chronic myelogenous leukaemia. Haematoxylin-eosin, $\times 100$

больного $[11,12]$. К настоящему времени в литературе описано немногим более 30 наблюдений развития обоих заболеваний у одного больного, в 13 из них ММ и ХМЛ диагностированы одномоментно (табл. 1). У 11 из 33 больных, включая настоящее клиническое наблюдение, первично была диагностирована ММ, у 10 больных изначально был диагностирован ХМЛ. Обращает на себя внимание примерно равное количество больных в каждой когорте, что ставит под сомнение теорию возникновения второго заболевания (ХМЛ либо ММ) в результате терапии первого заболевания. В большинстве клинических наблюдений при диагностике ХМЛ выявлен химерный ген $B C R-A B L$ (р210). В приведенном клиническом наблюдении с помощью FISH были выявлены сразу два варианта химерного гена: $B C R-A B L$ (p190) и $B C R-A B L(\mathrm{p} 210)$, что ранее не было описано. Анализ эффективности вариантов проведенной терапии показал преимущество применения ингибиторов протеасом (бортезомиба) и иммуномодуляторов (талидомида, леналидомида) при лечении ММ и ИТК (иматиниба, нилотиниба, дазатиниба) - при лечении ХМЛ. Выбранная схема лечения, включавшая ингибиторы протеасом и иммуномодуляторы, позволила достичь иммунохимической ремиссии ММ и сохранять ее на протяжении 3 лет, а применение дазатиниба привело к достижению большого молекулярного ответа. Однако в результате проводимой терапии дазатинибом и талидомидом у больной в течение длительного времени сохранялась изолированная тромбоцитопения. Прогрессия ХМЛ и рецидив ММ не отразились на гемограмме: концентрации лейкоцитов, гемоглобина 


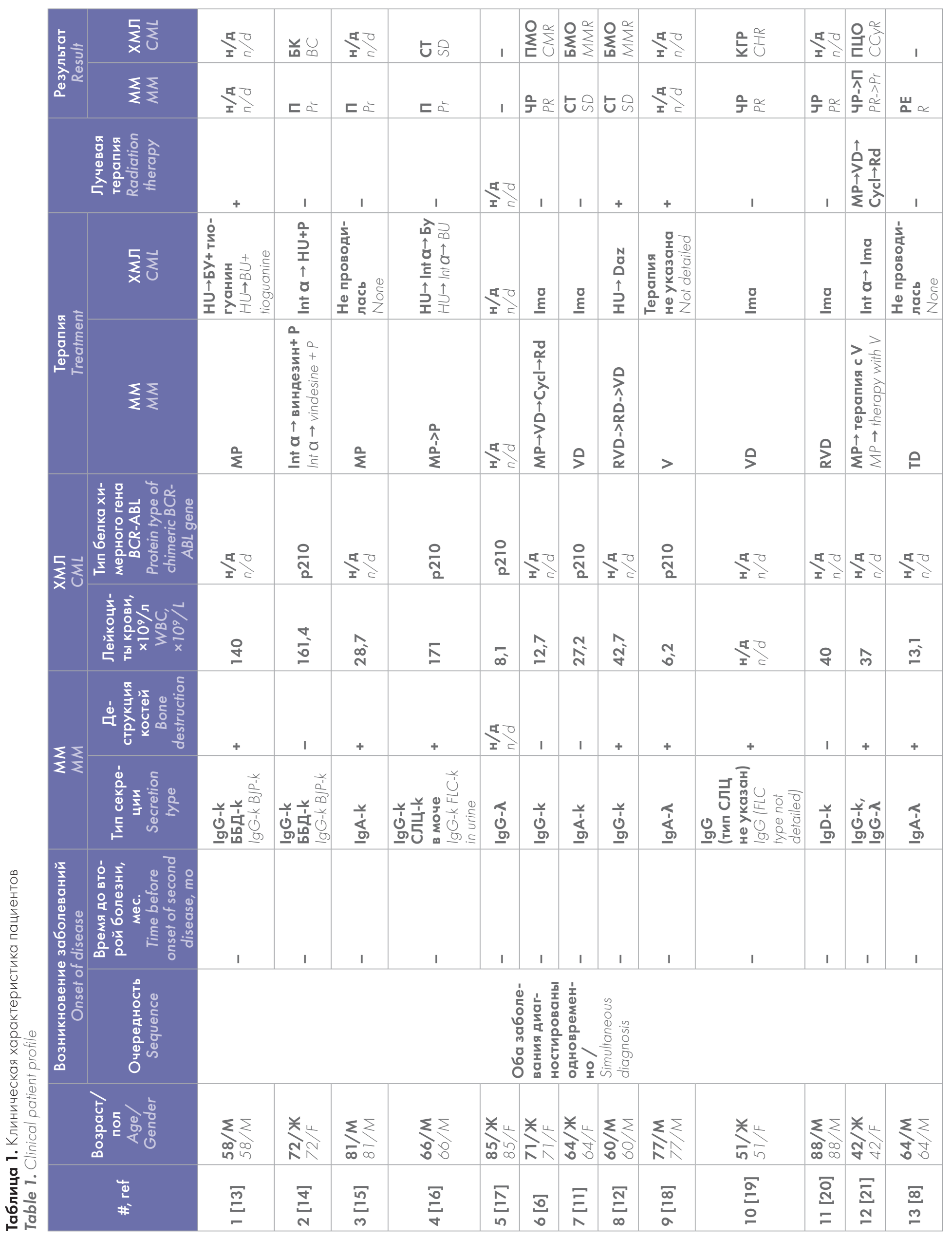




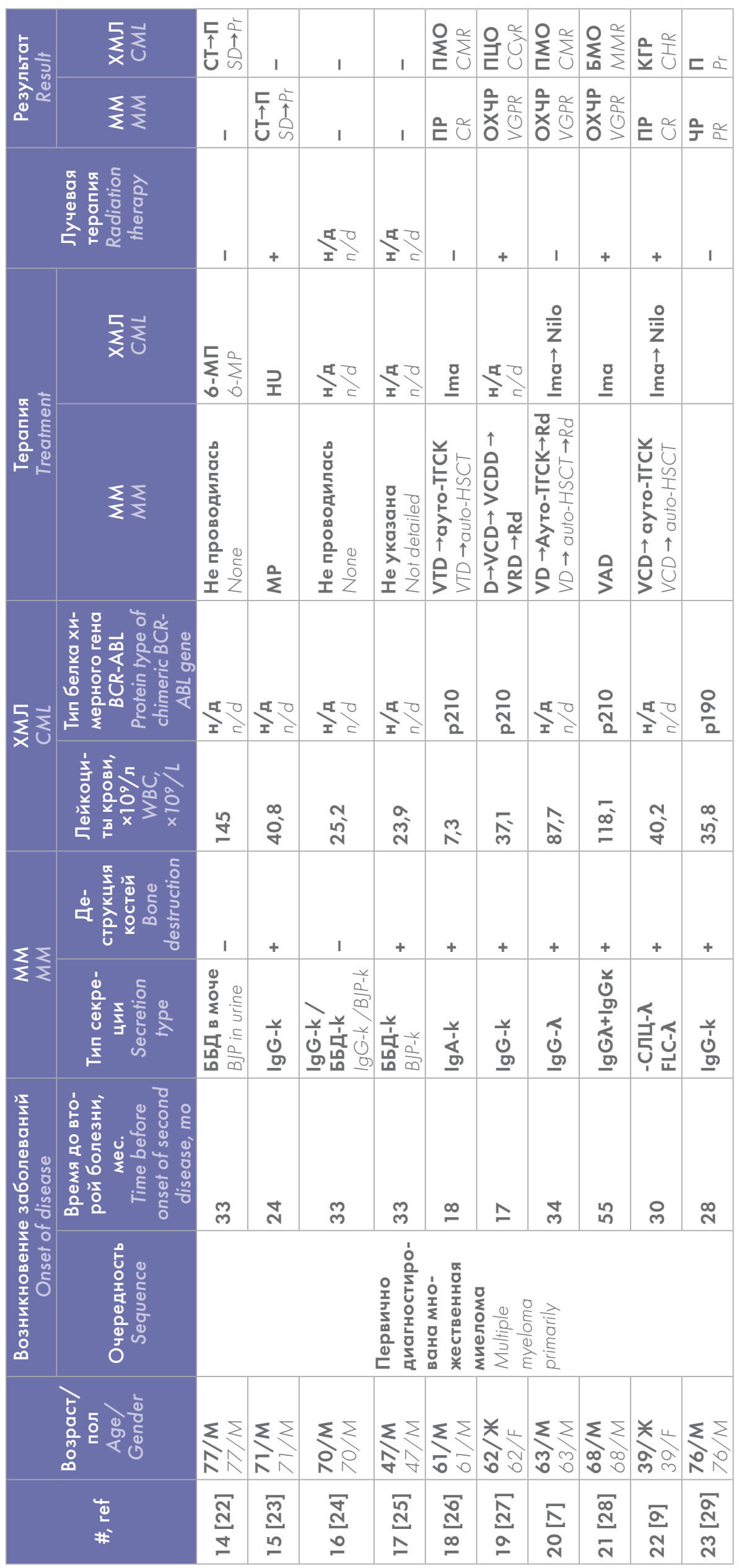




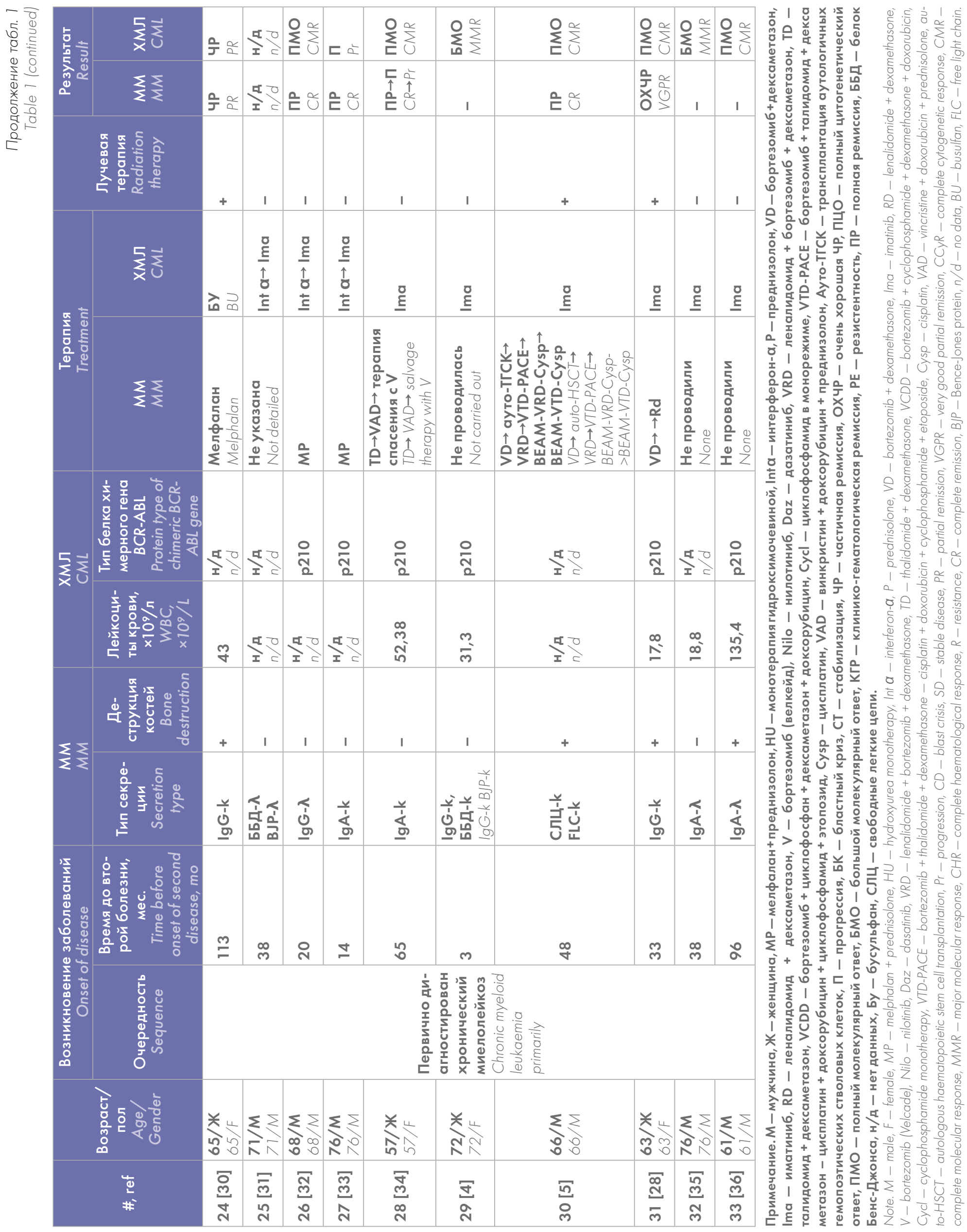


оставались в пределах нормы, тромбоцитопения не нарастала, что, возможно, было обусловлено либо ранней диагностикой рецидива и прогрессии заболеваний, либо закономерным следствием лечения. Однако для подтверждения данной гипотезы необходимо большее количество аналогичных клинических наблюдений.

Множество гипотез предложено для объяснения возникновения ХМЛ и ММ у одного больного. Одна из них основана на феномене клонального гемопоэза неопределенного потенциала, согласно которому существует плюрипотентная клетка-предшественница с возникшей одной или несколькими хромосомными аберрациями, например с транслокацией $\mathrm{t}(9 ; 22)$, способная к полноценной дифференцировке в миелоидные и лимфоидные линии [4, 21, 28, 29]. В нескольких клинических исследованиях [14, 23, 37] у больных ХМЛ филадельфийская хромосома определялась во всех клетках, включая мегакариоциты, макрофаги, клетки эритропоэза и иммуноглобулин-синтезирующие В-лимфоциты. Клональные В-лимфоциты при ХМЛ могут сохранять способность к дифференцировке до плазматических клеток [21].

Выявление химерного гена $B C R-A B L$ в одной из линий культуры клеток MM свидетельствует в пользу существования общей мутированной плюрипотентной клетки-предшественницы [38]. Генетические поломки при развитии ММ происходят в В-клетках герминального центра. С другой стороны, нельзя исключить возникновение мутаций и на ранних этапах дифференцировки клеток. Это предположение позволяет рассматривать развитие MМ в контексте теории двойной мутации Кнудсона [21].

У нескольких больных ММ был диагностирован сложный кариотип с многочисленными аберрациями, затрагивающими также хромосомы 9 и 22 [39, 40].

В более позднем наблюдении [16] FISH-исследование клеток костного мозга у больного с одновременным возникновением ХМЛ и ММ выявило полное отсутствие химерного гена $B C R-A B L$ в клональных плазматических клетках. В $100 \%$ клеток с $\mathrm{Ph}$-хромосомой отсутствовали мутации, выявленные позже в плазматических клетках. Применение молекулярно-генетических исследований при диагностике MМ и ХМЛ в дальнейших исследованиях [4, 27, 28, 34, 35] подтвердило результаты данного исследования. В настоящее время признано, что ХМЛ происходит из гемопоэтической стволовой клетки, а ММ - из В-клетки постгерминативного центра, что делает маловероятным, чтобы эти опухоли когда-либо имели общее клональное происхождение [26].

В нескольких клинических наблюдениях ссылаются на возможное существование причинно-следственной связи между приемом препаратов ИТК и развитием MM и, наоборот, влиянием препаратов, используемых для лечения MM, на заболеваемость ХМЛ [4, 7, 12, 18,
20, 27-29, 32, 33]. Иматиниб ингибирует клеточный цикл плазматических клеток in vitro путем взаимодействия с рецепторной тирозинкиназой III типа (с-KIT). Действие иматиниба препятствовало пролиферации атипичных плазматических клеток, резистентных к терапии дексаметазоном и/или мелфаланом, а также оказывало незначительное положительное влияние на деление плазматических клеток, опосредованно воздействуя на рецепторы $\operatorname{Erk} 1 / 2$, что могло спровоцировать развитие MM [41]. Однако неизвестно, оказывает ли влияние иматиниб на атипичные плазматические клетки in vivo [34]. A. Dispenzieri и соавт. [42] начали даже клиническое исследование по применению иматиниба для лечения больных рецидивирующей/рефрактерной MM, однако оно было прекращено, так как у большинства больных отмечена прогрессия MМ в результате проводимой терапии.

C другой стороны, в исследованиях L. J. Crawford и соавт. [43], а также О. Bucur и соавт. [44] было показано, что одновременное применение ингибиторов протеасом и ИТК позволяет преодолеть резистентность у Ph-позитивных клеток, резистентных к монотерапии иматинибом. Синергический эффект достигается путем взаимодействия с иммунопротеасомой опухолевых клеток. Это приводило к угнетению сигнального пути ERK и усилению экспрессии белка ABI $1 \frac{1}{2}$ и кофактора Cdh 1 в анафазе, в результате чего активировался апоптоз и ингибировалась пролиферация клеток-мишеней.

Известно, что применение леналидомида является фактором риска возникновения вторичных злокачественных заболеваний кожи и миелодиспластического синдрома. Однако в настоящее время не существует достоверных сведений, что терапия леналидомидом может привести к развитию ХМЛ [45]. Возникновение вторичного ХМЛ, в отличие от вторичных острых лейкозов, - крайне редкое явление. Возможно, это объясняется «индолентной природой» клетки-предшественницы ХМЛ, что делает ее менее восприимчивой к повреждению ДНК вследствие воздействия химиопрепаратов [27].

Изучена роль ионизирующего излучения в возникновении ХМЛ на примере людей, переживших взрыв атомной бомбы в Японии, а также больных, получавших лучевую терапию [46]. Рассчитано [46, 47], что после 1 Гр лучевой терапии в одной плюрипотентной стволовой клетке вероятность возникновения филадельфийской хромосомы по отношению к остальным хромосомным аберрациям составляет $7 \times 10^{-12}$. Принимая во внимание тот факт, что количество стволовых клеток у человека в среднем составляет $1 \times 10^{9}$ [48], вероятность возникновения транслокации $\mathrm{t}(9 ; 22)$ в одной стволовой клетке у одного человека составляет 0,7\% [46]. Следовательно, существует вероятность развития вторичного ХМЛ в результате лучевой терапии ММ. 
Медленно прогрессирующее течение ММ приводит к нарушению эффективной работы иммунной системы и, как следствие, формируется благоприятное микроокружение («ниша») для формирования новых злокачественных заболеваний. Клональные плазматические клетки также могут инициировать формирование вторичных лейкозов/лимфом посредством воздействия на многочисленные потенциальные профили экспрессии генов и молекулярные пути [49].

Существует еще множество теорий, объясняющих одновременно возникновение ММ и ХМЛ у одного больного. К факторам риска развития относят даже пол, возраст, образ жизни и окружающую среду. Тем

\section{Литература}

1. Поддубная И.В., Савченко В.Г. Российские клинические рекомендации по диагностике лечению лимфопролиферативных заболеваний. М., 2019.

2. American Cancer Society. Cancer Facts \& Figures 2020. Atlanta, Ga: American Cancer Society, 2020.

3. Jabbour E., Kantariian H. Chronic myeloid leukemia: 2018 update on diagnosis, therapy and monitoring. Am J Hematol. 2018; 93: 442-59. DOI: 10.1002/ajh.25011. 4. Ide M., Kuwahara N., Matsuishi E. et al. Uncommon case of chronic myeloid leukemia with multiple myeloma. Int J Hematol. 2010; 91: 699-704. DOI: 10.1007/s12185-010-0546-4.

5. Guru Murthy G.S., Sawyer J., Alapat D. et al. Strange bedfellows: mitotically active chronic myeloid leukemia in molecular complete remission, detected in focal lesion of myeloma. Clin Lymphoma Myeloma Leuk. 2014; 14: e127-9. DOI: 10.1016/j.clml.2014.02.008.

6. Offiah C., Quinn J.P., Thornton P., Murphy P.T. Coexisting chronic myeloid leukaemia and multiple myeloma: rapid response to lenalidomide during imatinib treatment. Int J Hematol. 2012; 95(4): 451-2. DOI: 10.1007/s12185-012-1038-5.

7. Katzel J.A., Lee-Ma A., Vesole D.H. Safety of a second-generation tyrosine kinase inhibitor and novel targeted therapy for the treatment of a patient with chronic myeloid leukemia and multiple myeloma. Anti-Cancer Drugs. 2015; 26(8): 907-9. DOI: 10.1097/CAD.0000000000000262.

8. Lee J.Y., Lee S.M., Yoon H.K. et al. A case of synchronous multiple myeloma and chronic myeloid leukemia. Blood Res. 2017; 52(3): 219-22. DOI: 10.5045/ br.2017.52.3.219.

9. Barrett A., Glavey S., Sargent J. Emergence of chronic myeloid leukemia following autologous stem cell transplantation in a young woman with multiple myeloma. Blood Res. 2018; 53(3): 254-6. DOI: 10.5045/br.2018.53.3.254.

10. Бессельцев С.С., Карягина Е.В., Стельмашенко Л.В. и др. Частота, характеристика и методы лечения периферической нейропатии и больных множественной миеломой, получающих бортезомиб (велкейд). Онкогематология. 2008; 3: 52-62.

11. Романенко Н.А., Бессмельцев С.С., Удальева В.Ю. и др. Сочетание хронического миелолейкоза и множественной миеломы у одного пациента. Вопросы онкологии. 2013; 59(2): 103-10.

12. Alsidawi S., Ghose A., Qualtieri J., Radhakrishnan N. A case of multiple myeloma with metachronous chronic myeloid leukemia treated successfully with bortezomib, dexamethasone, and dasatinib. Case Rep Oncol Med. 2014; 2014 : 962526. DOI: 10.1155/2014/962526.

13. Boots M.A., Pegrum G.D. Simultaneous presentation of chronic granulocytic leukaemia and multiple myeloma. J Clin Pathol. 1982; 35: 364-5. DOI: 10.1136/ icp.35.3.364 не менее, влияние ни одного из них к настоящему времени не доказано [18].

Таким образом, возникновение ММ и ХМЛ - событие, которое редко возникаету одного больного. Описано лишь несколько подобных случаев. Настоящее наблюдение выявило наличие сразу двух вариантов химерного гена $B C R-A B L(\mathrm{p} 190)$ и $B C R-A B L(\mathrm{p} 210)$. Предложено несколько гипотез, объясняющих возникновение обоих заболеваний у одного больного, однако ни одна из них не получила подтверждения. Это позволяет предположить, что одновременное развитие обоих заболеваний может быть следствием влияния множества факторов либо случайным и редким событием.

\section{References}

1. Poddubnaya I.V., Savchenko V.G. Russian clinical recommendations on diagnosis and treatment of lymphoproliferative diseases. Moscow, 2018; 306 (In Russian).

2. American Cancer Society. Cancer Facts \& Figures 2020. Atlanta, Ga: American Cancer Society, 2020.

3. Jabbour E., Kantariian H. Chronic myeloid leukemia: 2018 update on diagnosis, therapy and monitoring. Am J Hematol. 2018; 93: 442-59. DOI: 10.1002/ajh.25011. 4. Ide M., Kuwahara N., Matsuishi E. et al. Uncommon case of chronic myeloid leukemia with multiple myeloma. Int J Hematol. 2010; 91: 699-704. DOI: 10.1007/s12185-010-0546-4.

5. Guru Murthy G.S., Sawyer J., Alapat D. et al. Strange bedfellows: mitotically active chronic myeloid leukemia in molecular complete remission, detected in focal lesion of myeloma. Clin Lymphoma Myeloma Leuk. 2014; 14: e127-9. DOI: 10.1016/j.clml.2014.02.008.

6. Offiah C., Quinn J.P., Thornton P., Murphy P.T. Coexisting chronic myeloid leukaemia and multiple myeloma: rapid response to lenalidomide during imatinib treatment. Int J Hematol. 2012; 95(4): 451-2. DOI: 10.1007/s12185-012-1038-5.

7. Katzel J.A., Lee-Ma A., Vesole D.H. Safety of a second-generation tyrosine kinase inhibitor and novel targeted therapy for the treatment of a patient with chronic myeloid leukemia and multiple myeloma. Anti-Cancer Drugs. 2015; 26(8): 907-9. DOI: 10.1097/CAD.0000000000000262.

8. Lee J.Y., Lee S.M., Yoon H.K. et al. A case of synchronous multiple myeloma and chronic myeloid leukemia. Blood Res. 2017; 52(3): 219-22. DOI: 10.5045/ br.2017.52.3.219.

9. Barrett A., Glavey S., Sargent J. Emergence of chronic myeloid leukemia following autologous stem cell transplantation in a young woman with multiple myeloma. Blood Res. 2018; 53(3): 254-6. DOI: 10.5045/br.2018.53.3.254.

10. Bessmeltsev S.S., Karyagina E.V., Stelmashenko L.V. et al. Incidence, properties and therapy of peripheral neuropathy and multiple myeloma in patients receiving bortezomib (Velcade). Onkogematologiya. 2008; 3: 52-62. (In Russian).

11. Romanenko N.A., Bessmeltsev S.S., Udalyeva V.Y. et al. Co-occurrence of chronic myeloid leukaemia and multiple myeloma in one patient. Voprosi Onkologii. 2013; 59: 103-10 (In Russian).

12. Alsidawi S., Ghose A., Qualtieri J., Radhakrishnan N. A case of multiple myeloma with metachronous chronic myeloid leukemia treated successfully with bortezomib, dexamethasone, and dasatinib. Case Rep Oncol Med. 2014; 2014 962526. DOI: 10.1155/2014/962526.

13. Boots M.A., Pegrum G.D. Simultaneous presentation of chronic granulocytic leukaemia and multiple myeloma. J Clin Pathol. 1982; 35: 364-5. DOI: 10.1136/ icp.35.3.364. 
14. Tanaka M., Kimura R., Matsutani A. et al. Coexistence of chronic myelogenous leukemia and multiple myeloma. Case report and review of the literature. Acta Haematol. 1998; 99: 221-3. DOI: 10.1159/000040843.

15. Alvarez-Larrán A., Rozman M., Cervantes F. Simultaneous occurrence of multiple myeloma and chronic myeloid leukemia. Haematologica. 2001; 86: 894.

16. Schwarzmeier J.D., Shehata M., Ackermann J. et al. Simultaneous occurrence of chronic myeloid leukemia and multiple myeloma: evaluation by FISH analysis and in vitro expansion of bone marrow cells. Leukemia. 2003; 17: 1426-8. DOI: 10.1038/sj.leu.2402971.

17. Wakayama T., Fujita S., Ago H. An elder patient coexisted with multiple myeloma and chronic myeloid leukemia before treatments. Med J Shimane Pref Cent Hosp. 2005; 29: 63-7.

18. Maerki J., Katava G., Siegel D. et al. Unusual case of simultaneous presentation of plasma cell myeloma, chronic myelogenous leukemia, and a jak2 positive myeloproliferative disorder. Case Rep Hematol. 2014; 2014: 738428. DOI: $10.1155 / 2014 / 738428$.

19. Wolleschak D., Heidel F.H. Chronic myelogenous leukemia evolving after treatment of multiple myeloma. Blood. 2016; 128(1): 146. DOI: 10.1182/ blood-2016-03-706945

20. Ali N., Pickens P.V., Auerbach H.E. Immunoglobulin D multiple myeloma, plasma cell leukemia and chronic myelogenous leukemia in a single patient treated simultaneously with lenalidomide, bortezomib, dexamethasone and imatinib. Hematology Reports. 2016; 8(1): 6295. DOI: 10.4081/hr.2016.6295.

21. Рыжко В.В., Грачев А.Е., Клодзинский А.А. и др. Миелопролиферативные «маски» множественной миеломы (обзор литературы и описание клинических наблюдений). Терапевтический архив. 2017; 89(1): 72-7. DOI: 10.17116/terarkh201789172-77.

22. MacSween J.M., Langley G.R. Light-chain disease (hypogammaglobulinemia and Bence Jones proteinurial and sideroblastic anemia-preleukemic chronic granulocytic leukemia. Can Med Assoc J. 1972; 106: 995-8.

23. Klenn P.J., Hyun B.H., Lee Y.H., Zheng W.Y. Multiple myeloma and chronic myelogenous leukemia a case report with literature review. Yonsei Med J. 1993; 34: 293-300. DOI: 10.3349/ymi.1993.34.3.293.

24. Nitta M., Tsuboi K., Yamashita S. et al. Multiple myeloma preceding the development of chronic myelogenous leukemia. Int J Hematol. 1999; 69(3): $170-3$.

25. Nakagawa M., Noto S., Kobayashi H., Hayashi M. A case of a 47 year old man who developed chronic myelogenous leukemia after therapy for multiple myeloma. J Obihiro Kosei Gen Hosp. 2003; 6: 101-6.

26. Roper N., DeAngelo D.J., Kuo F. et al. An asymptomatic 61-year-old man with BCR ABL-positive bone marrow following autologous transplantation for multiple myeloma. Am J Hematol. 2010; 85(12): 944-6. DOI: 10.1002/ajh.21809.

27. Ragupathi L., Najfeld V., Chari A. et al. A case report of chronic myelogenous leukemia in a patient with multiple myeloma and a review of the literature. Clin Lymphoma Myeloma Leuk. 2013; 13(2): 175-9. DOI: 10.1016/i.clml.2012.09.010. 28. Pessach I., Bartzis V., Tzenou T. et al. Multiple myeloma and chronic myelogenous leukemia; an uncommon coexistence in 2 patients, with literature review. Ann Hematol Oncol. 2015; 2(3): 1030.

29. Miki K., Obara N., Makishima K. et al. An unprecedented case of pl90 BCR-ABL chronic myeloid leukemia diagnosed during treatment for multiple myeloma: A case report and review of the literature. Case Rep Hematol. 2018; 2018 : 7863943. DOI: 10.1155/2018/7863943.

30. Derghazarian C., Whittemore N.B. Multiple myeloma superimposed on chronic myelocytic leukemia. Can Med Assoc J. 1974; 110: 1047-50.

31. Yokota A, Onoda M, Uehara T, Terano T. Coexistence of chronic myelogenous leukemia and multiple myeloma. Rinsho Ketsueki. 2005; 46: 919.
14. Tanaka M., Kimura R., Matsutani A. et al. Coexistence of chronic myelogenous leukemia and multiple myeloma. Case report and review of the literature. Acta Haematol. 1998; 99: 221-3. DOI: 10.1159/000040843.

15. Alvarez-Larrán A., Rozman M., Cervantes F. Simultaneous occurrence of multiple myeloma and chronic myeloid leukemia. Haematologica. 2001; 86: 894.

16. Schwarzmeier J.D., Shehata M., Ackermann J. et al. Simultaneous occurrence of chronic myeloid leukemia and multiple myeloma: evaluation by FISH analysis and in vitro expansion of bone marrow cells. Leukemia. 2003; 17: 1426-8. DOI: 10.1038/sj.leu.2402971.

17. Wakayama T., Fujita S., Ago H. An elder patient coexisted with multiple myeloma and chronic myeloid leukemia before treatments. Med J Shimane Pref Cent Hosp. 2005; 29: 63-7.

18. Maerki J., Katava G., Siegel D. et al. Unusual case of simultaneous presentation of plasma cell myeloma, chronic myelogenous leukemia, and a jak2 positive myeloproliferative disorder. Case Rep Hematol. 2014; 2014: 738428. DOI: $10.1155 / 2014 / 738428$

19. Wolleschak D., Heidel F.H. Chronic myelogenous leukemia evolving after treatment of multiple myeloma. Blood. 2016; 128(1): 146. DOI: 10.1182/ blood-2016-03-706945.

20. Ali N., Pickens P.V., Auerbach H.E. Immunoglobulin D multiple myeloma, plasma cell leukemia and chronic myelogenous leukemia in a single patient treated simultaneously with lenalidomide, bortezomib, dexamethasone and imatinib. Hematology Reports. 2016; 8(1): 6295. DOI: 10.4081/hr.2016.6295.

21. Ryzhko V.V., Grachev A.E., Klodzinsky A.A., et al. Myeloproliferative masks of multiple myeloma: a review and clinical reports. Terapevticheskiy arkhiv. 2017; 89(1): 72-7. DOI: 10.17116/terarkh201789172-77. (In Russian).

22. MacSween J.M., Langley G.R. Light-chain disease (hypogammaglobulinemia and Bence Jones proteinuria) and sideroblastic anemia-preleukemic chronic granulocytic leukemia. Can Med Assoc J. 1972; 106: 995-8.

23. Klenn P.J., Hyun B.H., Lee Y.H., Zheng W.Y. Multiple myeloma and chronic myelogenous leukemia a case report with literature review. Yonsei Med J. 1993; 34: 293-300. DOI: 10.3349/ymi.1993.34.3.293.

24. Nitta M., Tsuboi K., Yamashita S. et al. Multiple myeloma preceding the development of chronic myelogenous leukemia. Int J Hematol. 1999; 69(3): $170-3$.

25. Nakagawa M., Noto S., Kobayashi H., Hayashi M. A case of a 47 year old man who developed chronic myelogenous leukemia after therapy for multiple myeloma. J Obihiro Kosei Gen Hosp. 2003; 6: 101-6.

26. Roper N., DeAngelo D.J., Kuo F. et al. An asymptomatic 61-year-old man with BCR ABL-positive bone marrow following autologous transplantation for multiple myeloma. Am J Hematol. 2010; 85(12): 944-6. DOI: 10.1002/ajh.21809.

27. Ragupathi L., Najfeld V., Chari A. et al. A case report of chronic myelogenous leukemia in a patient with multiple myeloma and a review of the literature. Clin Lymphoma Myeloma Leuk. 2013; 13(2): 175-9. DOI: 10.1016/i.clml.2012.09.010. 28. Pessach I., Bartzis V., Tzenou T. et al. Multiple myeloma and chronic myelogenous leukemia; an uncommon coexistence in 2 patients, with literature review. Ann Hematol Oncol. 2015; 2(3): 1030.

29. Miki K., Obara N., Makishima K. et al. An unprecedented case of p190 BCR-ABL chronic myeloid leukemia diagnosed during treatment for multiple myeloma: A case report and review of the literature. Case Rep Hematol. 2018; 2018: 7863943. DOI: 10.1155/2018/7863943.

30. Derghazarian C., Whittemore N.B. Multiple myeloma superimposed on chronic myelocytic leukemia. Can Med Assoc J. 1974; 110: 1047-50.

31. Yokota A, Onoda M, Uehara T, Terano T. Coexistence of chronic myelogenous leukemia and multiple myeloma. Rinsho Ketsueki. 2005; 46: 919. 
32. Garipidou V., Vakalopoulou S., Tziomalos K. Development of multiple myeloma in a patient with chronic myeloid leukemia after treatment with imatinib mesylate. Oncologist. 2005; 10(6): 457-8. DOI: 10.1634/theoncologist.10-6-457. 33. Galanopoulos A., Papadhimitriou S.I., Kritikou-Griva E. et al. Multiple myeloma developing after imatinib mesylate therapy for chronic myeloid leukemia. Ann Hematol. 2009; 88: 281-2. DOI: 10.1007/s00277-008-0597-2.

34. Michael M., Antoniades M., Lemesiou E. et al. Development of multiple myeloma in a patient with chronic myeloid leukemia while on treatment with imatinib mesylate for 65 months. Oncologist. 2009; 14: 1198-1200. DOI: 10.1634/theoncologist.2009-0165.

35. Ahn S., Park J.S., Han J.H., Cho S.R. A rare case of chronic myelogenous leukemia and plasma cell myeloma in the same patient. Ann Lab Med. 2015; 35(3): 370-2. DOI: 10.3343/alm.2015.35.3.370.

36. Lee J.H., Kim Y.K. Plasma cell myeloma in a patient with chronic myelogenous leukemia. Lab Med Online. 2017; 7(1): 49-51. DOl: org/10.3343/ Imo.2017.7.1.49.

37. Martin P.J., Najfeld V., Hansen J.A. et al. Involvement of the B-lymphoid system in chronic myelogenous leukaemia. Nature. 1980; 287: 49-50. DOI: 10.1038/287049aO.

38. Breitkopf S.B., Yuan M., Pihan G.A., Asara J.M. Detection of a rare BCR-ABL tyrosine kinase fusion protein in $\mathrm{H} 929$ multiple myeloma cells using immunoprecipitation (IP)-tandem mass spectrometry (MS/MS). Proc Natl Acad Sci USA. 2012; 109(40): 16190-5. DOI: 10.1073/pnas.1212759109.

39. Van Den Berghe H., Louwagie A., Broeckaert-Van Orshoven A. et al. Philadelphia chromosome in human multiple myeloma. J Natl Cancer Inst. 1979; 63: 11-16. 40. Martiat P., Mecucci C., Nizet Y. et al. P190 BCR/ABL transcript in a case of Philadelphia-positive multiple myeloma. Leukemia. 1990; 4: 751-4.

41. Pandiella A., Carvajal-Vergara X., Tabera S. et al. Imatinib mesylate (STI571) inhibits multiple myeloma cell proliferation and potentiates the effect of common antimyeloma agents. BrJ Haematol. 2003; 123: 858-68. DOI: 10.1046/i.13652141.2003.04706.x

42. Dispenzieri A., Gertz M.A., Lacy M.Q. et al. A phase II trial of imatinib in patients with refractory/relapsed myeloma. Leuk Lymphoma. 2006; 47(1): 39-42. DOI: 10.1080/10428190500271269.

43. Crawford L.J., Chan E.T., Aujay M. et al. Synergistic effects of proteasome inhibitor carfilzomib in combination with tyrosine kinase inhibitors in imatinib-sensitive and -resistant chronic myeloid leukemia models. Oncogenesis. 2014; 3(3); 1-10. DOI: 10.1038/oncsis.2014.3.

44. Bucur O., Stancu A.L., Goganau I. et al. Combination of bortezomib and mitotic inhibitors down-modulate Bcr-Abl and efficiently eliminates tyrosine-kinase inhibitor sensitive and resistant Bcr-Abl-positive leukemic cells. PLoS One. 2013; 8(10): e77390. DOI: 10.1371/journal.pone.0077390.

45. Dimopoulos M.A., Richardson P.G., Brandenburg N. et al. A review of second primary malignancy in patients with relapsed or refractory multiple myeloma treated with lenalidomide. Blood. 2012; 119(12): 2764-7. DOI: 10.1182/ blood-2011-08-373514

46. Little M.P., Weiss H.A., Boice J.D. et al. Risks of leukemia in Japanese atomic bomb survivors, in women treated for cervical cancer, and in patients treated for ankylosing spondylitis. Radiat Res. 1999; 152(3): 280-92.

47. Holmberg M. Is the primary event in radiation-induced chronic myelogenous leukemia the induction of the $t(9 ; 22)$ translocation? Leuk Res. 1992; 16: 333-6.

48. Clinical Hematology. 8th Edition. Eds M.M. Wintrobe et al. Lea and Febiger, Philadelphia, 1981.

49. Thomas A., Mailankody S., Korde N. et al. Second malignancies after multiple myeloma: From 1960s to 2010s. Blood. 2012; 119(12): 2731-7. DOI: 10.1182/blood-2011-12-381426.
32. Garipidou V., Vakalopoulou S., Tziomalos K. Development of multiple myeloma in a patient with chronic myeloid leukemia after treatment with imatinib mesylate. Oncologist. 2005; 10(6): 457-8. DOI: 10.1634/theoncologist.10-6-457. 33. Galanopoulos A., Papadhimitriou S.I., Kritikou-Griva E. et al. Multiple myeloma developing after imatinib mesylate therapy for chronic myeloid leukemia. Ann Hematol. 2009; 88: 281-2. DOI: 10.1007/s00277-008-0597-2.

34. Michael M., Antoniades M., Lemesiou E. et al. Development of multiple myeloma in a patient with chronic myeloid leukemia while on treatment with imatinib mesylate for 65 months. Oncologist. 2009; 14: 1198-1200. DOI: 10.1634/theoncologist.2009-0165.

35. Ahn S., Park J.S., Han J.H., Cho S.R. A rare case of chronic myelogenous leukemia and plasma cell myeloma in the same patient. Ann Lab Med. 2015; 35(3): 370-2. DOI: 10.3343/alm.2015.35.3.370.

36. Lee J.H., Kim Y.K. Plasma cell myeloma in a patient with chronic myelogenous leukemia. Lab Med Online. 2017; 7(1): 49-51. DOI: org/10.3343/ Imo.2017.7.1.49.

37. Martin P.J., Najfeld V., Hansen J.A. et al. Involvement of the B-lymphoid system in chronic myelogenous leukaemia. Nature. 1980; 287: 49-50. DOI: 10.1038/287049aO.

38. Breitkopf S.B., Yuan M., Pihan G.A., Asara J.M. Detection of a rare BCR-ABL tyrosine kinase fusion protein in $\mathrm{H} 929$ multiple myeloma cells using immunoprecipitation (IP)-tandem mass spectrometry (MS/MS). Proc Natl Acad Sci USA. 2012; 109(40): 16190-5. DOI: 10.1073/pnas.1212759109.

39. Van Den Berghe H., Louwagie A., Broeckaert-Van Orshoven A. et al. Philadelphia chromosome in human multiple myeloma. J Natl Cancer Inst. 1979; 63: 11-16. 40. Martiat P., Mecucci C., Nizet Y. et al. P190 BCR/ABL transcript in a case of Philadelphia-positive multiple myeloma. Leukemia. 1990; 4: 751-4.

41. Pandiella A., Carvajal-Vergara X., Tabera S. et al. Imatinib mesylate (STI571) inhibits multiple myeloma cell proliferation and potentiates the effect of common antimyeloma agents. Br J Haematol. 2003; 123: 858-68. DOI: 10.1046/i.13652141.2003.04706.x.

42. Dispenzieri A., Gertz M.A., Lacy M.Q. et al. A phase II trial of imatinib in patients with refractory/relapsed myeloma. Leuk Lymphoma. 2006; 47(1): 39-42. DOI: 10.1080/10428190500271269.

43. Crawford L.J., Chan E.T., Aujay M. et al. Synergistic effects of proteasome inhibitor carfilzomib in combination with tyrosine kinase inhibitors in imatinib-sensitive and -resistant chronic myeloid leukemia models. Oncogenesis. 2014; 3(3); 1-10. DOI: 10.1038/oncsis.2014.3.

44. Bucur O., Stancu A.L., Goganau I. et al. Combination of bortezomib and mitotic inhibitors down-modulate Bcr-Abl and efficiently eliminates tyrosine-kinase inhibitor sensitive and resistant Bcr-Abl-positive leukemic cells. PLoS One. 2013; 8(10): e77390. DOI: 10.1371/journal.pone.0077390.

45. Dimopoulos M.A., Richardson P.G., Brandenburg N. et al. A review of second primary malignancy in patients with relapsed or refractory multiple myeloma treated with lenalidomide. Blood. 2012; 119(12): 2764-7. DOI: 10.1182/ blood-2011-08-373514.

46. Little M.P., Weiss H.A., Boice J.D. et al. Risks of leukemia in Japanese atomic bomb survivors, in women treated for cervical cancer, and in patients treated for ankylosing spondylitis. Radiat Res. 1999; 152(3): 280-92.

47. Holmberg M. Is the primary event in radiation-induced chronic myelogenous leukemia the induction of the $+(9 ; 22)$ translocation? Leuk Res. 1992; 16: 333-6.

48. Clinical Hematology. 8th Edition. Eds M.M. Wintrobe et al. Lea and Febiger, Philadelphia, 1981.

49. Thomas A., Mailankody S., Korde N. et al. Second malignancies after multiple myeloma: From 1960s to 2010s. Blood. 2012; 119(12): 2731-7. DOI: 10.1182/blood-2011-12-381426. 


\section{Информация об авторах}

Ольга Валерьевна Рыбина", врач-гематолог отделения гематологии, ЧУЗ «Центральная клиническая больница «РЖД-Медицина», e-mail: Olia.fishka@yandex.ru, тел.: +7 (985) 267-20-12

ORCID: https://orcid.org/0000-0002-9531-1025

Юлия Александровна Шавель, врач-гематолог высшей квалификационной категории отделения гематологии, ЧУЗ «Центральная клиническая больница «РЖД-Медицина»,

e-mail:gematdoc@gmail.com

ORCID: https://orcid.org/0000-0003-4395-1968

Андрей Анатольевич Петренко, врач-гематолог, ГБУЗ г. Москвы Городская клиническая больница им. С. П. Боткина Департамента здравоохранения г. Москвы,

e-mail: petrenkoandrei13@gmail.com

ORCID: https://orcid.org/0000-0001-8461-5421

Мария Владимировна Галайко, врач-гематолог отделения гематологии, ЧУЗ «Центральная клиническая больница «РЖД-Медицина»,

e-mail: loima@mail.ru

ORCID: https://orcid.org/0000-0002-5375-0273

Мария Сергеевна Литвиненко, врач-гематолог отделения гематологии,

ЧУЗ «Центральная клиническая больница «РЖД-Медицина»,

e-mail: maryasergeevnal@yandex.ru

ORCID: https://orcid.org/0000-0001-7134-3893

Владимир Евгеньевич Егорков, врач-гематолог отделения гематологии, ЧУЗ «Центральная клиническая больница «РЖД-Медицина»,

e-mail: ckb2gem@mail.ru

ORCID: https://orcid.org/0000-0003-0298-072X

Андрей Владимирович Губкин, кандидат медицинских наук, главный гематолог Центральной дирекции здравоохранения-филиала ОАО «РЖД», заведующий отделением, ЧУЗ «Центральная клиническая больница «РЖДМедицина»,

e-mail: gubkinav@gmail.com

ORCID: https://orcid.org/0000-0003-3698-7751

* Автор, ответственный за переписку

Поступила: 18.02 .2020

Принята в печать: 27.10.2020

\section{Information about the authors}

Olga V. Rybina*, Haematologist, Department of Haematology, Central Clinical Hospital "RZD-Medicine",

e-mail: Olia.fishka@yandex.ru, tel.: +7 (985) 267-20-12

ORCID: https://orcid.org/0000-0002-9531-1025

Jylia A. Shavel, Haematologist (higher degree), Department of Haematology, Central Clinical Hospital "RZD-Medicine",

e-mail: gematdoc@gmail.com

ORCID: https://orcid.org/0000-0003-4395-1968

Andrei A. Petrenko, Haematologist, Botkin Moscow City Hospital,

e-mail: petrenkoandreil3@gmail.com

ORCID: https://orcid.org/0000-0001-8461-5421

Marya V. Galaiko, Haematologist, Department of Haematology, Central Clinical Hospital "RZD-Medicine",

e-mail: loima@mail.ru

ORCID: https://orcid.org/0000-0002-5375-0273

Marya S. Litvinenko, Haematologist, Department of Haematology, Central Clinical Hospital "RZD-Medicine",

e-mail: maryasergeevnal@yandex.ru

ORCID: https://orcid.org/0000-0001-7134-3893

Vladimir E. Egorkov, Haematologist, Department of Haematology, Central Clinical Hospital "RZD-Medicine",

e-mail: ckb2gem@mail.ru

ORCID: https://orcid.org/0000-0003-0298-072X

Andrey V. Gubkin, Cand. Sci. (Med.), Chief Haematologist, Central Healthcare Directorate-Branch of "OAO RZhD", Head of the Department of Haematology, Central Clinical Hospital "RZD-Medicine",

e-mail: gubkinav@gmail.com

ORCID: https://orcid.org/0000-0003-3698-7751

* Corresponding author

Received 18 Feb 2020

Accepted 27 Oct 2020 